\title{
PRÁTICAS COLABORATIVAS NA CADEIA DE SUPRIMENTOS E O PAPEL DAS NOVAS TECNOLOGIAS DE INFORMAÇÃO
}

\author{
Mariana Ribeiro de Castro ${ }^{1}$ \\ Marcelo Bronzo Ladeira²
}

\begin{abstract}
RESUMO
Diferentes teorias e modelos foram validados, nos últimos anos, em pesquisas orientadas à compreensão dos antecedentes, correlatos e consequentes de práticas colaborativas sobre o desempenho de empresas e cadeias de suprimento. Há também evidências empíricas que corroboram a importância das novas tecnologias de informação para o avanço de práticas colaborativas e para a integração de processos no contexto das cadeias de suprimentos. Este artigo, de natureza teórica e conceitual, procura sintetizar parte dessas contribuições e avançar, ao propor uma discussão sobre o papel das novas tecnologias de informação na promoção das práticas colaborativas, como um construto multidimensional, complexo e fortemente influenciado pela integração dos processos interorganizacionais.
\end{abstract}

Palavras-chave: Práticas colaborativas. Tecnologias de informação. Gestão da Cadeia de Suprimentos.

\footnotetext{
${ }^{1}$ Graduada, e-mail: marianacastro18@yahoo.com.br

2Doutor, e-mail: marcelobronzo@face.ufmg.br
} 


\section{INTRODUÇÃO}

A gestão efetiva da cadeia de suprimentos tem se tornado um meio potencialmente valioso de assegurar vantagem competitiva e melhorar o desempenho organizacional. Nesse sentido, o interesse das empresas em perseguir níveis superiores de serviços e empreender práticas avançadas de gerenciamento de suas cadeias de suprimentos (sob a forma de parcerias estratégicas com fornecedores, customização de processos e relacionamento com clientes, planejamento colaborativo de demanda, maior intensidade no compartilhamento de informações estratégicas sobre produtos e mercados, qualidade das informações compartilhadas e adiamento da configuração final de produtos, por exemplo) podem levar a incrementos de performance e a definir formas sustentáveis de vantagens competitivas para empresas e suas respectivas cadeias (ATTARAN; ATTARAN, 2007; Ll et al., 2006).

Sheffi (2002) afirma que uma das maneiras mais eficientes para melhorar a acurácia das previsões de venda e aumentar o nível de serviço ao cliente, reduzindo custos ao mesmo tempo, é melhorar a colaboração entre parceiros comerciais. Pires (2004, p. 164) define colaboração, no contexto da literatura em gestão da cadeia de suprimentos, como "relacionamentos de longo prazo entre empresas que trabalham juntas e integradas na busca de objetivos comuns". Segundo o autor, a colaboração na cadeia de suprimentos geralmente ocorre quando duas ou mais empresas dividem a responsabilidade de trocar informações acerca do planejamento, gestão, execução e medição de desempenho.

Para Attaran e Attaran (2007), a ausência ou deficiência no planejamento colaborativo pode ter um impacto significativo no desempenho da cadeia de suprimentos. Os autores citam um estudo da AMA Research, empresa especializada na publicação de relatórios, boletins e dados de mercado, em que vem corroborada a premissa de que a colaboração na cadeia de suprimentos pode aumentar cerca de três pontos percentuais as margens de lucro para todos os agentes na cadeia de suprimentos. Segundo Cao e Zhang (2011), empresas como a Hewlett-Packard, IBM, Dell e Procter \& Gamble investiram em um relacionamento colaborativo de longo prazo com seus fornecedores para reduzir custos de transação e garantir um posicionamento competitivo mais forte. Relacionamentos colaborativos podem ajudar as empresas a compartilhar riscos, acessar recursos complementares, reduzir custos de transação, aumentar a produtividade, aumentar o desempenho em lucro e a vantagem competitiva ao longo do tempo (CAO; ZHANG, 2011; MENTZER et al., 2001).

Power (2005) comenta que a integração dos sistemas na gestão da cadeia de suprimentos tem sido objeto de significantes debates e discussão. Como as organizações têm buscado desenvolver relacionamentos e integrar informações de forma mais efetiva com parceiros comerciais, os processos internos tendem a se tornar interligados e extrapolar as fronteiras tradicionais das empresas. Cria-se, assim, uma maior dependência de tecnologias de informação, que se tornam viabilizadoras de novos arranjos cooperativos. As empresas passam a ser vistas como uma rede de processos, relacionamentos e tecnologias, aumentando sua interdependência e o compartilhamento de objetivos. 
No contexto dos processos interorganizacionais e dos fluxos de valor entre empresas, Seggie, Kim e Cavusgil (2006) propõem, com base na teoria da visão baseada em recursos de Barney (1991), que a integração dos sistemas interempresariais e o alinhamento dos recursos de tecnologia de informação entre membros do canal tornam-se fontes de vantagem competitiva sustentável para as empresas.

Corroborando com esta proposta, Pramatari (2007) declara quea tecnologia de informação tem desempenhado um papel facilitador nas práticas colaborativas, ressaltando que há uma clara evolução nas capacidades e sofisticação da infraestrutura de tecnologia de informação quando se compara as antigas e as novas formas de colaboração. As implementações iniciais de parcerias comerciais eletrônicas focaram a automação das transações utilizando o Intercâmbio Eletrônico de Dados (Electronic Data Interchange - EDI). Outras práticas e iniciativas a serviço do planejamento e gestão colaborativa entre parceiros comerciais, especialmente no âmbito da cadeia de suprimentos, foram difundidas no mercado, tais como o Estoque Gerenciado pelo Fornecedor (Vendor ManagedInventory - VMI), o Programa de Ressuprimento Contínuo (Continuous Replenishment Program - CRP) e a Resposta Eficiente ao Consumidor (Efficient Consumer Response - ECR) (ATTARAN; ATTARAN, 2007; PIRES, 2004; PRAMATARI, 2007).

O objetivo central deste artigo, de cunho teórico-conceitual, é o de apresentar a síntese de um levantamento bibliográfico cujo propósito foi o de delinear variáveis e construtos relevantes na condição de antecedentes, correlatos e consequentes do uso da tecnologia de informação na implementação de práticas colaborativas em cadeias de suprimentos. Em um primeiro momento, são abordados elementos analíticos e mainstreams teóricos dos quais é possível inferir utilidade para a prática da pesquisa sobre o tema, tais como: a teoria baseada em recursos (RBV), a teoria dos custos de transação, a teoria de redes. Em um segundo momento, são discutidas no artigo as potenciais dimensões de colaboração nos processos interorganizacionais nas redes de suprimento e, então, apresentada uma síntese de modelos e teorias que referenciam as novas tecnologias de informação como condição não somente desejável mas absolutamente necessária à integração do fluxo de valor no contexto das cadeias de suprimento. Seguem as conclusões do artigo, com uma síntese das ideias discutidas no texto bem como a proposição de temas para futuros estudos sobre o tema.

\section{TEORIAS E ELEMENTOS ANALÍTICOS ÚTEIS AO ESTUDO DA COLABORAÇÃO NO CONTEXTO DAS CADEIAS DE SUPRIMENTOS}

Há escassez de pesquisas que contemplem a natureza multidimensional da colaboração no contexto das cadeias de suprimentos (WIENGARTEN et al., 2010). De acordo com Simatupang e Sridharan (2002), praticantes e acadêmicos estão crescentemente interessados na colaboração na cadeia de suprimentos, nos fatores que viabilizam sua implementação e nos fatores críticos para seu sucesso. Nesse sentido, diversos autores defendem que as práticas colaborativas devem incorporar a tomada de decisão conjunta 
e o alinhamento de incentivos, sendo o compartilhamento efetivo de informações, um fator essencial para viabilizar a incorporação destes processos nas práticas entre parceiros comerciais (LEEUW; FRANSOO, 2009; SEGGIE; KIM; CAVUSGIL; 2006; SIMATUPANG; SRIDHARAN, 2002).

Quadro 1: Lista construtos de relacionamentos na cadeia de suprimentos e integração na literatura

\begin{tabular}{|c|c|c|}
\hline Referência & $\begin{array}{l}\text { Conceituação de } \\
\text { relacionamentos comprador- } \\
\text { fornecedor }\end{array}$ & Resultados principais \\
\hline $\begin{array}{l}\text { Cannon e } \\
\text { Perreault } \\
(1999)\end{array}$ & $\begin{array}{l}\text { Relacionamentos comprador- } \\
\text { fornecedor são manifestas em: } \\
\text { trocas de informação, ligação } \\
\text { operacional, laços legais, normas } \\
\text { cooperativas, adaptações por } \\
\text { vendedores e compradores. }\end{array}$ & $\begin{array}{l}\text { Oito tipos de relacionamentos } \\
\text { foram desenvolvidos, cada um } \\
\text { provendo evidência da diversidade } \\
\text { como compradores e fornecedores } \\
\text { conduzem os negócios. }\end{array}$ \\
\hline $\begin{array}{l}\text { Stanket al. } \\
(2001)\end{array}$ & $\begin{array}{l}\text { Colaboração é definida como um } \\
\text { processo de tomada de decisão } \\
\text { entre partes interdependentes, } \\
\text { que envolve decisões conjuntas } \\
\text { e responsabilidade coletiva pelos } \\
\text { resultados. }\end{array}$ & $\begin{array}{l}\text { Resultados indicaram que a } \\
\text { colaboração interna é associada } \\
\text { com níveis mais altos de } \\
\text { desempenho em serviço logístico, } \\
\text { enquanto a colaboração externa } \\
\text { não possui esta associação. }\end{array}$ \\
\hline $\begin{array}{l}\text { Frohlich e } \\
\text { Westbrook } \\
(2001)\end{array}$ & $\begin{array}{l}\text { Conceituaram integração na } \\
\text { cadeia de suprimentos através da } \\
\text { direção (em relação a clientes e/ } \\
\text { ou fornecedores) e extensão da } \\
\text { integração. }\end{array}$ & $\begin{array}{l}\text { Resultados indicaram que quanto } \\
\text { maior o grau de integração com } \\
\text { fornecedores e clientes, maiores as } \\
\text { melhorias em desempenho. }\end{array}$ \\
\hline $\begin{array}{l}\text { Vickeryet al. } \\
(2003)\end{array}$ & $\begin{array}{l}\text { Integração na cadeia de } \\
\text { suprimentos conceituada através } \\
\text { de práticas que compreendem } \\
\text { integração tanto intrafirmas quanto } \\
\text { interfirmas. }\end{array}$ & $\begin{array}{l}\text { Integração na cadeia de } \\
\text { suprimentos afeta positivamente o } \\
\text { serviço ao cliente e indiretamente o } \\
\text { desempenho financeiro através do } \\
\text { serviço ao cliente. }\end{array}$ \\
\hline $\begin{array}{l}\text { Simatupang } \\
\text { e Sridharan } \\
(2002)\end{array}$ & $\begin{array}{l}\text { Índice de colaboração na cadeia de } \\
\text { suprimentos é conceituado através } \\
\text { de alinhamento de incentivos, } \\
\text { compartilhamento de informação e } \\
\text { sincronização de decisão. }\end{array}$ & $\begin{array}{l}\text { Achados mostram que o índice de } \\
\text { colaboração afeta positivamente o } \\
\text { desempenho operacional. }\end{array}$ \\
\hline $\begin{array}{l}\text { Vereecke } \\
\text { e Muylle } \\
(2006)\end{array}$ & 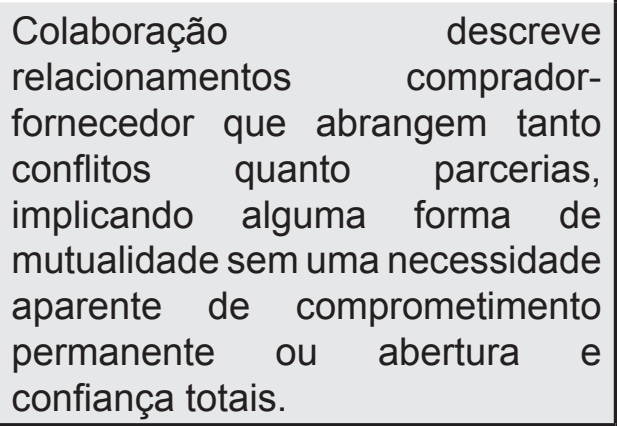 & $\begin{array}{l}\text { Colaboração somente melhora } \\
\text { marginalmente as taxas de } \\
\text { melhoria de desempenho. Troca de } \\
\text { informação melhora o desempenho } \\
\text { em termos de custo, flexibilidade, } \\
\text { qualidade e indicadores } \\
\text { relacionados a compras. }\end{array}$ \\
\hline
\end{tabular}


Leeuw e Fransoo (2009) declaram que muitos estudos enfocaram os efeitos da colaboração no desempenho, mas poucos enfocaram os fatores que influenciam a colaboração. Em relação especificamente ao fator tecnologia de informação no contexto da implementação de práticas colaborativas na cadeia de suprimentos, coexistem posicionamentos diversos na literatura concernentes à maturidade das tecnologias correntes em relação aos requisitos de colaboração e em que medida a tecnologia de informação pode ser um viabilizador ou uma barreira nas iniciativas colaborativas (PRAMATARI; 2007).

O propósito da gestão da cadeia de suprimentos, segundo Kaufman (1997), é remover as barreiras de comunicação e eliminar redundâncias através da coordenação, monitoramento e controle de processos. A integração da cadeia de suprimentos seria, assim, uma tentativa de consolidar as ligações entre cada componente da cadeia, melhorando o processo de tomada de decisão e fazendo com que todas as partes da cadeia interajam de modo mais efetivo (POWER, 2005). Simatupang e Sridharan (2002) afirmam que as empresas constituintes de uma cadeia de suprimentos se envolvem na gestão desta cadeia ao integrar o planejamento, a implementação e o controle dos fluxos de bens e serviços, e informações relacionadas, para o propósito de atender aos requisitos dos clientes. Desta forma, o relacionamento próximo e a cooperação ajudam os membros da cadeia de suprimentos a efetivamente equilibrarem demanda e oferta para aumentar a lucratividade de toda a cadeia. Uma cadeia de suprimentos colaborativa, segundo os autores, significa simplesmente que duas ou mais empresas independentes trabalham em conjunto para planejar e executar operações da cadeia com maior sucesso que alcançariam caso agissem de forma isolada.

O reconhecimento da importância dos relacionamentos interorganizacionais como fonte de vantagem competitiva e de criação de valor, especialmente no contexto da gestão da cadeia de suprimentos, tem crescido em vários campos associados à pesquisa organizacional (KRAUSE; HANDFIELD; TYLER, 2007; PAULRAJ; LADO; CHEN, 2008). Segundo Lavassani, Movehedi e Kumar (2008), até o início dos anos 2000, os esforços acadêmicos estiveram mais focados em conceituar e definir que em prover um arcabouço teórico para a gestão da cadeia de suprimentos. Algumas abordagens teóricas, entretanto, têm sido utilizadas nos estudos sobre gestão da cadeia de suprimentos e fundamentam o entendimento tanto da gestão da cadeia de suprimentos quanto dos relacionamentos colaborativos entre seus membros (LAVASSINI; MOVEHEDI; KUMAR, 2008; KETCHEN; HULT, 2007).

Segundo Lavassani, Movehedi e Kumar (2008), os pressupostos fundamentais à teoria dos custos de transação (TransactionCostTheory - TCT), muito utilizada na economia e nos estudos organizacionais, podem ser facilmente emprestados à análise de diferentes práticas de gestão e da colaboração na cadeia de suprimentos. Williamson (1985) sugere que as organizações podem reduzir seus custos de transação por meio da integração vertical e pelo aumento no nível de confiança. O estabelecimento de relações mais confiáveis entre empresas poderia reduzir a assimetria de informações acerca da transação e reduzir o risco de comportamentos oportunistas entre os parceiros. 
No entanto, esta perspectiva negligencia as oportunidades de geração de valor na transação que os relacionamentos interorganizacionais podem originar. Lavassani, Movehedi e Kumar (2008) ressaltam que as empresas em uma cadeia de suprimentos podem reduzir custos não somente pela integração vertical, mas também pela integração horizontal, com outras empresas membros da cadeia, e pela economia de escala alcançada pela agregação de oferta e/ou demanda.

Hashiba (2008) pondera que as empresas em uma cadeia podem maximizar o valor da transação ao atingir altos volumes nas negociações, compartilhar informações, desenvolver proteções auto-reforçadas e descobrir novas maneiras de melhorar o desempenho com o uso de ativos específicos, explorando as vantagens de eficiência em relação a redes menos especializadas, por exemplo. Segundo Zajac e Olsen (1993), o reconhecimento da interdependência dos parceiros na transação à procura de ganhos e o contexto do relacionamento e dos processos ao longo do tempo proporcionam uma análise mais consistente das estratégias interorganizacionais, complementando a abordagem da TCT.

Lavassani, Movehedi e Kumar (2008) comentam sobre a possibilidade de se estudar a gestão da cadeia de suprimentos e os relacionamentos interempresarias sob a perspectiva das teorias de rede. Os autores afirmam que esta perspectiva se preocupa com a estrutura e conteúdo das relações interorganizacionais, as quais podem aumentar as capabilidades em recursos e as competências de empresas individuais por meio da coordenação estratégica da rede. Neste sentido, Gameiro (2005) afirma que a aproximação entre os membros de uma rede, visando a colaboração e a criação de vantagens competitivas, faz com que as empresas tenham mais condições de satisfazer de maneira mais eficaz as necessidades do mercado em que atuam. Um melhor aproveitamento de recursos, a partilha de riscos e o fortalecimento das empresas são vistos por Gameiro (2005) como potenciais benefícios que podem advir do relacionamento cooperativo.

Já os pressupostos da Visão Baseada em Recursos (Resource Based View - RBV) parecem assumir que as empresas, podem ser heterogêneas em relação aos seus recursos estratégicos e que estes recursos, não sendo transferíveis facilmente entre as empresas, acabam por gerar heterogeneidade mais duradoura, gerando valor e limitando a competição. De acordo com a RBV, os recursos tangíveis e intangíveis da organização influenciam na criação e na sustentabilidade da vantagem competitiva da organização, sobretudo quando eles podem ser considerados valiosos, raros, difíceis de serem imitados e não substituíveis (BARNEY, 1991).

De acordo com as proposições de Dierickx e Cool (1989), a colaboração (e os seus resultados) pode ser observada como um recurso crítico, que só pode ser obtido através da sua construção. Hashiba (2008) afirma que a colaboração entre firmas e a capacidade de estabelecê-la são considerados recursos críticos da empresa por serem valiosas, raras e de difícil imitação. A colaboração pode ser considerada fonte de vantagem competitiva por não ocorrer automaticamente, tendo que superar barreiras e desenvolver capacidades organizacionais, que não são facilmente imitadas por outras empresas (BARRAT, 2004). 
Se na RBV as empresas são vistas como um conjunto de recursos, que são os fatores centrais para a formulação de suas estratégias e heterogeneidade de desempenho, a Visão Relacional (RelationaIView - RV) segue o mesmo princípio. A contribuição fundamental da RV é que ela delineou uma teoria para considerar díades e redes de empresas como uma unidade de análise chave para explicar o desempenho superior individual da empresa, enquanto a unidade de análise na RBV, por exemplo, era a empresa e seus recursos internos (DYER; SINGH, 1998). Segundo Hashiba (2008), a RV pode ser vista como uma extensão da RBV, já que o seu desenvolvimento não intenciona ou pressupõe ignorar a RBV, mas sim complementá-la, ao considerar os relacionamentos como recursos que oferecem vantagem competitiva, por serem valiosos, raros, insubstituíveis e inimitáveis.

Dyer e Singh (1998) afirmam que fontes de diferenças de desempenho e de lucratividade poderiam ser baseadas na rede estratégica de relacionamentos das empresas, sendo que a capacidade de integrar o conhecimento residente dentro e fora da empresa emerge como habilidade com potencial de diferenciá-la no mercado. Pela ótica da RV, recursos raros, valiosos, insubstituíveis e de difícil imitação são gerados também fora das empresas, nas suas fronteiras, incorporados ao relacionamento que estas organizações estabelecem. Neste sentido, Paulraj, Lado e Chen (2008) ponderam que, para as empresas, ter uma orientação de longo prazo é um fator essencial para formar, desenvolver e manter transações relacionais que gerem valor. Parceiros transacionais com esta orientação são propensos a apoiarem-se em normas de relações leais, capazes de originar benefícios significativos.

Paulraj, Lado e Chen (2008) afirmam que a RV provê um arcabouço teórico relevante para a investigação dos relacionamentos entre empresas na cadeia de suprimentos por algumas razões. Primeiro, pelo fato de a RV considerar o nível de análise interorganizacional e endereçar a extensão na qual capabilidades relacionais formam a base de vantagens estratégicas duráveis. Segundo, porque com base na RV é possível examinar como competências relacionais permitem às empresas obter e manter vantagens colaborativas.

Uma proposição central destas teorias é que quando as organizações investem em ativos relacionais específicos, se engajam em troca de conhecimento e informações e combinam recursos de forma colaborativa, um lucro superior pode ser obtido por ambas às partes da transação (KRAUSE; HANDFIELD; TYLER, 2007).

\section{DIMENSÕES DA COLABORAÇÃO}

Com o entendimento crescente de que a gestão da cadeia de suprimentos deveria ser construída em torno da colaboração entre parceiros comerciais, o tema ganhou relevância na literatura especializada (PRAMATARI, 2007; BARRAT; OLIVEIRA, 2001), em que termos como integração, colaboração, cooperação e coordenação são complementares em abordagens analíticas das cadeias de suprimentos (ARSHINDER; DESHMUKH, 2008; LEEUW; FRANSOO, 2009). 
Leeuw e Fransoo (2009) caracterizam a colaboração efetiva entre membros da cadeia de suprimentos pelos seguintes aspectos: (a) relacionamento de longo prazo entre organizações independentes; (b) atividades próximas cooperativas e coordenadas em aspectos como compartilhamento de informação, planejamento conjunto, gestão de demanda e gestão de estoque; (c) união de grupos distintos dentro e entre empresas; (d) objetivos comuns e compartilhados; (e) perspectiva compartilhada dos benefícios de laços estreitos; e (f) criação de visibilidade.

Segundo Power (2005), os níveis crescentes de competição global, a emergência de novos tipos de relacionamento interorganizacionais e a revolução nas formas de intercâmbio de informação foram os principais direcionadores para o estabelecimento das práticas colaborativas e integradoras nas cadeias de suprimento.

Autores como Ramanathan e Gunasekaran (2012) e Cao e Zhang (2011) definiram sete dimensões da colaboração interorganizacional: compartilhamento de informação; congruência de objetivos; sincronização de decisão; alinhamento de incentivos; compartilhamento de recursos; comunicação; e a criação conjunta de conhecimento. A fim de detalhar melhor os conceitos envolvidos nas dimensões da colaboração, ressaltando os aspectos críticos que se associam de forma mais direta à implementação da tecnologia de informação como suporte às práticas colaborativas, algumas dimensões merecem ser descritas com maior minúcia.

O compartilhamento de informação se refere à extensão na qual uma empresa compartilha uma variedade de informações relevantes, acuradas, completas e confidenciais em tempo hábil com seus parceiros na cadeia de suprimentos (CAO; ZHANG, 2011). Forslund e Jonsson (2007) descrevem o compartilhamento de informação como viabilizador essencial da colaboração na cadeia de suprimentos. Nyaga, Whipple e Lynch (2010), examinando relacionamentos nas perspectivas de compradores e fornecedores, provaram que o compartilhamento de informação colaborativo e os esforços e os investimentos conjuntos levaram à confiança e ao comprometimento dos parceiros comerciais. Cao e Zhang (2011) complementam a definição de compartilhamento de informação como a propensão em tornar dados estratégicos e táticos tais como níveis de estoque, previsões, promoções de venda, estratégias e estratégias de marketing disponíveis para as empresas que constituem os nós na cadeia de suprimentos.

Simatupang e Sridharan (2002) definem a sincronização de decisão como o processo pelo qual os parceiros da cadeia de suprimentos orquestram decisões no planejamento e operações da cadeia de suprimentos que otimizam benefícios na cadeia de suprimentos. Decisões de planejamento são requeridas para determinar a forma mais eficiente e efetiva de usar os recursos da empresa para atingir um conjunto específico de objetivos. O planejamento conjunto é usado para alinhar parceiras colaborativas e tomar decisões operacionais incluindo a reposição de estoque, colocação de pedidos e entrega de pedidos (CAO; ZHANG, 2011). O Planejamento, Previsão e Ressuprimento Colaborativos (Collaborative Planning, Forescastand Replenishment - CPFR), cuja implementação é fortemente facilitada pela utilização de recursos tecnológicos, especialmente a internet, 
é uma prática colaborativa que enfatiza o aspecto da sincronização de decisão entre parceiros comerciais (ATTARAN; ATTARAN, 2007).

Segundo Cao e Zhang (2011), o compartilhamento de recursos se refere ao processo de alavancagem de capacidades e ativos e ao investimento em capacidades e ativos com parceiros na cadeia de suprimentos. Os autores destacam que estes recursos incluem recursos físicos, tais como equipamentos de manufatura, instalações e tecnologia, e especificamente tecnologias de informação. No setor de varejo, por exemplo, práticas como o Estoque Gerenciado pelo Fornecedor (VendorManagedInventory - VMI) permitem aos fornecedores avaliar os dados de nível de estoque de seus clientes e tomar a ação de ressuprimento necessária no momento correto, favorecendo os níveis de serviço aos clientes (SEIFERT, 2003). Este tipo de prática exige a troca de informações com agilidade e qualidade, fator crítico para seu sucesso, e as tecnologias de informação podem exercer papel fundamental em seu funcionamento (PRAMATARI, 2007).

Cao e Zhang (2011) mencionam também a comunicação colaborativa, que é o contato e o processo de transmissão de mensagens entre parceiros da cadeia de suprimentos em termos de frequência, direção, modo e estratégia de influência. Comunicação aberta, frequente, bilateral e multinível é geralmente uma indicação de relacionamentos interorganizacionais estreitos. Esta dimensão da colaboração também pode ser fortemente impactada pela implementação bem sucedida de sistemas integrados de informação, que podem viabilizar novos canais de comunicação (RAMANATHAN; GUNASEKARAN, 2012).

Power (2005) resume os fatores essenciais para o sucesso de práticas colaborativas como o compartilhamento de informação, a confiança, a parceria, a tecnologia compartilhada e uma mudança fundamental de funções organizacionais individuais para a gestão de cadeias de processos integradas. Para que isso ocorra, sistemas de informação, fluxos de material e relacionamentos entre parceiros devem ser considerados elementos críticos de um modelo de cadeia de suprimentos integrada.

\section{NOVAS TECNOLOGIAS DE INFORMAÇÃO NA IMPLEMENTAÇÃO DE PRÁTICAS COLABORATIVAS}

Pramatari (2007) declara que a tecnologia de informação tem desempenhado um papel facilitador nas práticas colaborativas, ressaltando que há uma clara evolução nas capacidades e sofisticação da infraestrutura de tecnologia de informação quando se compara as antigas e as novas formas de colaboração. As implementações iniciais de parcerias comerciais eletrônicas focaram a automação das transações utilizando o Intercâmbio Eletrônico de Dados (Electronic Data Interchange - EDI). Outras práticas e iniciativas a serviço do planejamento e gestão colaborativa entre parceiros comerciais, especialmente no âmbito da cadeia de suprimentos, foram difundidas no mercado, tais como o Estoque Gerenciado pelo Fornecedor (Vendor Managed Inventory - VMI), 
oPrograma de Ressuprimento Contínuo (Continuous Replenishment Program - CRP) e a Resposta Eficiente ao Consumidor (Efficient Consumer Response - ECR) (ATTARAN; ATTARAN; 2007; PIRES, 2004; PRAMATARI; 2007).

A emergência de novas tecnologias, como a Identificação por Rádio Frequência (Radio Frequencyldentification - RFID), e a difusão de tecnologias associadas à internet potencializaram as práticas colaborativas na gestão da cadeia de suprimentos e geraram a expectativa de revolucionar muitas de suas operações, especialmente se o escopo da implementação for estendido de processos internos para processos da cadeia de suprimentos envolvendo parceiros (PRAMATARI, 2007). Um relatório da indústria aborda novos processos de colaboração que serão potencializados através do uso de RFID e do compartilhamento intensivo de informações entre parceiros comerciais, tais como o recall de produtos, a logística reversa e a gestão da validade de produtos $(\mathrm{GCl}, 2005)$. A Figura 1 mostra um exemplo de aplicação da RFID no atendimento de pedidos e seu potencial de uso em outras práticas colaborativas de uma cadeia de suprimentos.

Figura 1: Aplicação de RFID na gestão da cadeia de suprimentos

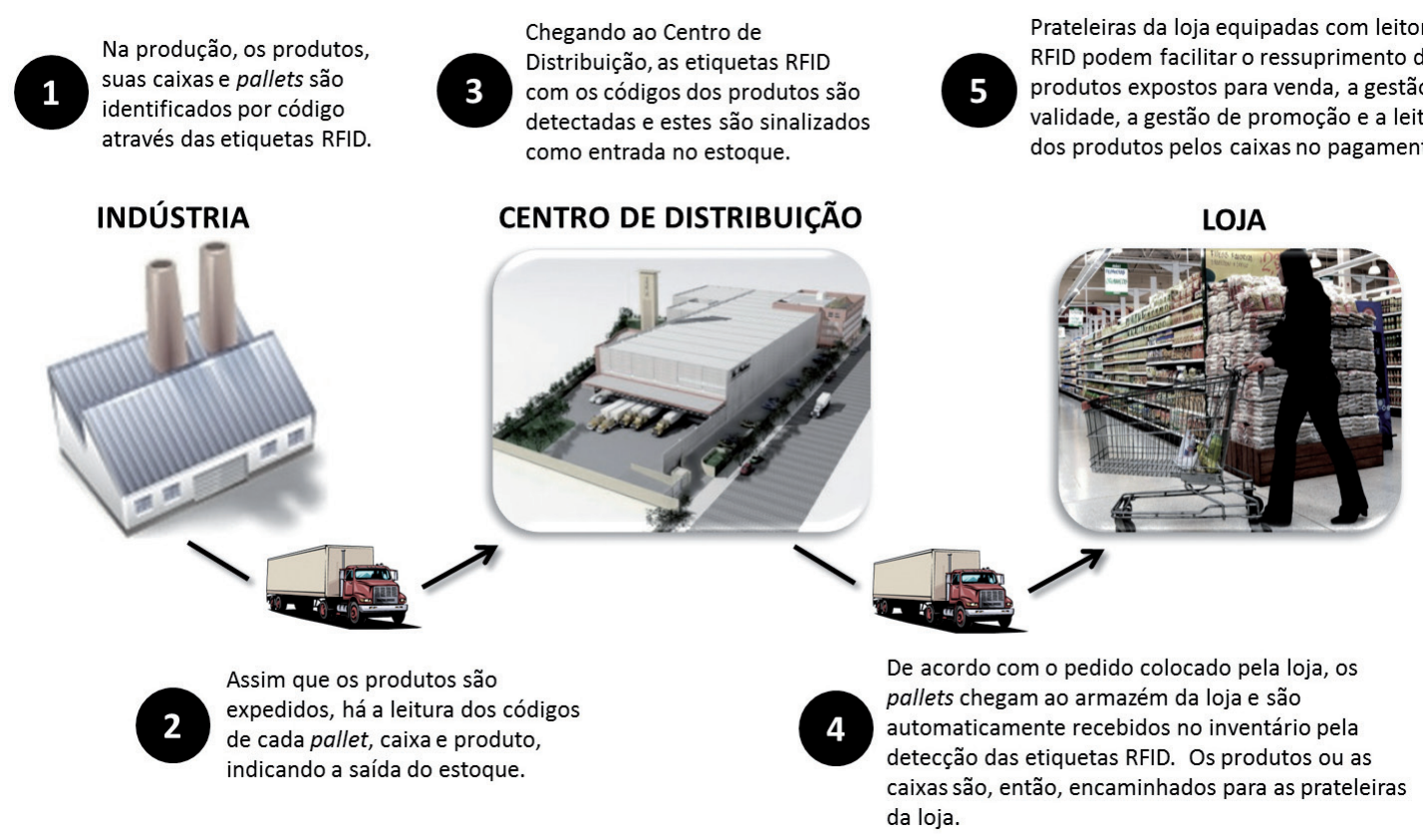

Fonte: Dos autores

Segundo Ramanathan e Gunasekaran (2012), a maioria dos artigos discutidos na literatura descreve os benefícios da colaboração na cadeia de suprimentos, mas falham em aprofundar essa discussão através de uma visão compreensiva dos fatores críticos para o sucesso da mesma. Wiengarten et al. (2010) afirmam que o impacto das práticas colaborativas na cadeia de suprimentos, tais como o compartilhamento de informação, 
o alinhamento de incentivos e a tomada de decisão conjunta, no desempenho das empresas varia significativamente dependendo da qualidade e da rapidez em que a informação é trocada entre os parceiros, levantando um indicativo de que a tecnologia de informação pode influenciar fortemente essa relação.

Bowersox, Closs e Stank (1999) afirmam que a integração na cadeia de suprimentos está largamente articulada em torno de tecnologia de informação avançada, já que a conectividade de sistemas interempresariais através de tal tecnologia determina quão efetivas as atividades de coordenação interempresariais são. Corroborando com esta noção, Leeuw e Fransoo (2009) argumentam que o alinhamento da tecnologia de informação com parceiros da cadeia de suprimentos e a existência de sistemas interempresariais sugerem a existência de capacidades tecnológicas para a realização de atividades efetivas na cadeia de suprimentos. A integração entre os parceiros, por possibilitar a coordenação eficiente entre empresas, possibilita a redução de custos de coordenação e pode ser crítica para servir os clientes com eficiência e eficácia (SEGGIE; KIM; CAVUSGIL, 2006).

De acordo com Power (2005), a evolução no conceito de integração levou ao entendimento de que a cadeia de suprimentos opera como uma entidade corporativa, abrangendo diversas empresas sem a referência dos limites individuais tradicionais. $O$ alinhamento de objetivos entre estas empresas deve estar embasado em cooperação e colaboração, sob o risco de haver um alinhamento pobre e prejudicial, como tradicionalmente vem ocorrendo entre as funções de produção e de vendas dentro das empresas. A integração de fato ocorre quando clientes e fornecedores estabelecem parcerias estreitas com os objetivos e prováveis resultados de redução de estoque, menores lead times e melhor serviço ao consumidor, estabelecendo o alinhamento como pré-condição para melhoria nas práticas de gestão da cadeia de suprimentos. Power e Sohal (2002) argumentam, ainda, que a mais efetiva destas cadeias será aquela que for capaz de obter o mix correto de requisitos de informação, logística física e colaboração, provendo benefícios compartilhados para a maioria das organizações parceiras.

Power (2005) declara que a aplicação efetiva da tecnologia de informação à integração das atividades da cadeia de suprimentos tem o efeito de redução nos níveis de complexidade. Como os métodos convencionais de previsão, planejamento e análise não são equipados para lidar com a complexidade dinâmica, quando os efeitos de intervenções não são óbvios ao longo do tempo (SENGE, 1990 apud POWER, 2005), a gestão efetiva da cadeia de suprimentos, apoiada largamente pela tecnologia de informação, ganha uma importância estratégica para as empresas.

Segundo o Voluntary Interindustry Commerce Standards (2004), a colaboração encoraja todos os atores da cadeia de suprimentos a se engajarem no planejamento, previsão, ressuprimento, compartilhamento de informação, compartilhamento de recursos e compartilhamento de incentivos. Empresas como a West-Marine, Procter \& Gamble e Hewlett-Packard provaram os benefícios da colaboração na cadeia de suprimentos em termos de redução de custos, melhoria em vendas e melhoria na acurácia de previsões, 
tornando o compartilhamento de benefícios o elemento chave de tais colaborações (RAMANATHAN; GUNASEKARAN, 2012). Algumas práticas gerenciais colaborativas como o VMI, ECR e CRP foram sugeridos na literatura para melhorar os fluxos materiais e de informação nas cadeias de suprimento. (SEIFERT, 2003).

As novas tecnologias de informação tem claramente desempenhado um papel facilitador em muitas, senão em todas, as práticas colaborativas nos contextos das cadeias de suprimentos, e para muitos os elementos tecnológicos são antecedentes e viabilizam tais práticas (PRAMATARI, 2007; SEGGIE; KIM; CAVUSGIL, 2006). O Quadro 2, abaixo, apresenta potenciais aplicações relevantes da TI no contexto da colaboração em redes de suprimentos.

Quadro 2: Informações compartilhadas e tecnologias subjacentes do processo de colocação de pedido tradicional ao processo colaborativo viabilizado por RFID

\begin{tabular}{|c|c|c|c|c|}
\hline & \multicolumn{4}{|c|}{ Prática colaborativa na cadeia de suprimentos } \\
\hline & $\begin{array}{l}\text { Processo de } \\
\text { colocação } \\
\text { de pedido } \\
\text { tradicional }\end{array}$ & VMI/CRP & CPFR & $\begin{array}{c}\text { Processo colaborativo } \\
\text { viabilizado por RFID }\end{array}$ \\
\hline $\begin{array}{l}\text { Informação } \\
\text { trocada }\end{array}$ & $\begin{array}{l}\text { Pedidos } \\
\text { Avisos de } \\
\text { despacho }\end{array}$ & $\begin{array}{l}\text { Pedidos } \\
\text { Pedidos } \\
\text { sugeridos } \\
\text { Relatório de } \\
\text { estoque } \\
\text { (incluindo } \\
\text { pedidos de } \\
\text { loja e } \\
\text { embarques } \\
\text { de armazéns em } \\
\text { CRP) }\end{array}$ & $\begin{array}{l}\text { Pedidos } \\
\text { Relatórios de } \\
\text { estoque } \\
\text { Dados de pontos } \\
\text { de venda agregados } \\
\text { Previsões de venda } \\
\text { Planos de } \\
\text { promoção }\end{array}$ & $\begin{array}{l}\text { Posição de } \\
\text { produto na } \\
\text { prateleira } \\
\text { Indicação } \\
\text { de produtos } \\
\text { faltantes } \\
\text { Informação } \\
\text { acurada de } \\
\text { estoque } \\
\text { (incluindo } \\
\text { estoque de } \\
\text { fundo de loja, } \\
\text { datas de validade, } \\
\text { etc.) } \\
\text { Dados de pontos } \\
\text { de venda } \\
\text { contextualizados } \\
\text { Histórico do produto }\end{array}$ \\
\hline
\end{tabular}




\begin{tabular}{|c|c|c|c|c|}
\hline $\begin{array}{l}\text { Processos } \\
\text { de negócio } \\
\text { colaborativos }\end{array}$ & Ressuprimento & $\begin{array}{l}\text { Ressuprimento } \\
\text { de Armazém } \\
\text { Central } \\
\text { (Central } \\
\text { Warehouse } \\
\text { - CWH) }\end{array}$ & $\begin{array}{l}\text { Ressuprimento } \\
\text { CWH } \\
\text { Ressuprimento } \\
\text { de loja } \\
\text { Planos de } \\
\text { promoção }\end{array}$ & $\begin{array}{l}\text { Ressuprimento } \\
\text { Anti-falsificação } \\
\text { Compliance legal } \\
\text { Recall de produtos } \\
\text { Logística reversa } \\
\text { Gestão de validade } \\
\text { de produtos } \\
\text { Monitoramento } \\
\text { de cadeias frias } \\
\text { Gestão de prateleira } \\
\text { Gestão de promoção }\end{array}$ \\
\hline $\begin{array}{l}\text { Tecnologia } \\
\text { suportando } \\
\text { a troca de } \\
\text { informação }\end{array}$ & $\begin{array}{l}\text { Papel } \\
\text { EDI } \\
\text { EDI através } \\
\text { da internet } \\
\text { Intermediação } \\
\text { via internet }\end{array}$ & $\begin{array}{l}\text { EDI } \\
\text { (principalmente) } \\
\text { EDI } \\
\text { através da } \\
\text { internet } \\
\text { (recentemente) } \\
\text { Intermediação } \\
\text { via internet }\end{array}$ & $\begin{array}{l}\text { Arquivos XML/ASCII } \\
\text { através da internet } \\
\text { Rede de } \\
\text { sincronização } \\
\text { de dados globais } \\
\text { (Global Data } \\
\text { Synchronization } \\
\text { Network } \\
\text { - GDSN) (esperado) }\end{array}$ & $\begin{array}{l}\text { Arquivos XML através } \\
\text { da internet (esperado) } \\
\text { Rede de código } \\
\text { eletrônico de produto } \\
\text { (ElectronicProductCode } \\
\text { - EPC) - Infraestrutura } \\
\text { de serviço de nome de } \\
\text { objeto (ObjectName } \\
\text { Service - ONS) } \\
\text { (esperado) } \\
\text { Rede de sincronização } \\
\text { de dados globais } \\
\text { (Global data } \\
\text { synchronization } \\
\text { network - GDSN) } \\
\text { (esperado) }\end{array}$ \\
\hline
\end{tabular}




\begin{tabular}{|c|c|c|c|c|}
\hline $\begin{array}{l}\text { Tecnologia } \\
\text { suportando } \\
\text { a interação } \\
\text { com o } \\
\text { usuário }\end{array}$ & $\begin{array}{l}\text { Sistemas ERP } \\
\text { internos }\end{array}$ & $\begin{array}{l}\text { Aplicação } \\
\text { interna } \\
\text { (principalmente) } \\
\text { Plataforma } \\
\text { colaborativa } \\
\text { - trocas de } \\
\text { varejo } \\
\text { (recentemente) }\end{array}$ & $\begin{array}{l}\text { Plataforma } \\
\text { colaborativa - } \\
\text { trocas de varejo }\end{array}$ & $\begin{array}{l}\text { Ambiente de } \\
\text { distribuição } \\
\text { distribuída } \\
\text { - serviços web } \\
\text { (esperado) } \\
\text { Plataforma } \\
\text { colaborativa } \\
\text { - trocas de varejo } \\
\text { (esperado) }\end{array}$ \\
\hline
\end{tabular}

Fonte: Adaptado de Pramatari (2007)

Com a emergência da internet, novos e até então inesperados canais para o compartilhamento de informação tornaram-se disponíveis para os membros das cadeias de suprimento (RAMANATHAN; GUNASEKARAN, 2012). O aumento de complexidade das informações nas cadeias de suprimento, a crescente sofisticação na tecnologia de informação e a possibilidade de utilização da internet criaram condições para o surgimento de uma nova prática colaborativa ou ferramenta para a gestão da cadeia de suprimentos: o Planejamento, Previsão e Ressuprimento Colaborativos (Collaborative Planning, Forecastingand Replenishment- CPFR). Esta prática explora as redes computacionais, a tecnologia de informação e outras tecnologias baseadas na internet, evidenciando que a evolução na tecnologia de informação acaba por permitir um avanço em relação às práticas colaborativas no contexto da cadeia de suprimentos (SEIFERT, 2003; VOLUNTARY INTERINDUSTRY COMMERCE STANDARDS, 2004).

Neste sentido, Pramatari (2007) argumenta que, com a passagem do VMI e CRP para formas mais avançadas de colaboração, tais como CPFR, a quantidade de informação intercambiada entre partes aumentou, assim como os requisitos em relação à infraestrutura tecnológica subjacente. O Intercâmbio Eletrônico de Dados (Electronic Data Interchange - EDI) foi uma tecnologia facilitadora chave para o ressuprimento eficiente e coordenação da cadeia de suprimentos. Sem o EDI, o CRP não teria sido economicamente viável, já que a quantidade de informação diária processada e transmitida no canal é muito grande para ser tratada de forma manual. A tecnologia de informação foi uma condição necessária para a inovação CRP, servindo, assim, como um viabilizador para esta nova forma de relacionamento interorganizacional e para o redesenho de processos conjuntos no canal (LEE; PAK; LEE, 2003).

Entretanto, o EDI também é caro e bastante complicado quando comparado às alternativas que surgiram posteriormente para a comunicação segura e confiável na 
internet e para a troca de informação no formato XML (ExtensibleMarkupLanguage) (PRAMATARI, 2007). Segundo Lee, Pak e Lee (2003), o desenvolvimento da linguagem XML e do EDI/XML (um padrão aberto para o envio das transmissões EDI pela internet) permitiu que as conexões entre os diferentes sistemas de informação das organizações se tornassem mais efetivas em custo e fáceis de implementar (ver Quadro 3). Isso porque a linguagem XML oferece um padrão flexível, escalável e que exige significativamente menos conhecimento especialista para a troca de informação entre parceiros comerciais via internet, estabelecendo a capacidade de troca de informações ricas a um custo comparativamente baixo.

Quadro 3: Comparação entre a abordagem EDI clássica e a abordagem baseada na Web para suportar processos de colocação de pedido e faturamento eletrônicos

\begin{tabular}{|c|c|c|c|}
\hline & & Abordagem EDI clássica & Abordagem baseada na Web \\
\hline \multirow{3}{*}{ 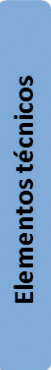 } & $\begin{array}{l}\text { Conformidade com } \\
\text { padrões }\end{array}$ & $\begin{array}{l}\text { Processo complicado e difícil. Empresas precisam } \\
\text { customizar padrões, entendê-los e controlar o } \\
\text { mapeamento entre dados internos e mensagens EDI }\end{array}$ & $\begin{array}{l}\text { Nenhum padrão comum exigido. Empresas enviam e } \\
\text { recebem informações em qualquer formato. } 0 \text { provedor de } \\
\text { serviço intermediário assume responsabilidade de fazer } 0 \\
\text { mapeamento do formato de uma empresa para o de outra }\end{array}$ \\
\hline & $\begin{array}{l}\text { Uso de infraestrutura } \\
\text { de rede }\end{array}$ & $\begin{array}{l}\text { Exigência de que as empresas se conectassem a VAN } \\
\text { (Value Added Network), que adicionava } \\
\text { complexidade técnica ao processo }\end{array}$ & $\begin{array}{l}\text { Empresas usam a infraestrutura da internet, já utilizada } \\
\text { pelas empresas normalmente }\end{array}$ \\
\hline & $\begin{array}{l}\text { Instalação de } \\
\text { software }\end{array}$ & $\begin{array}{l}\text { Exigência de que as empresas instalassem softwares } \\
\text { de EDI especializados, normalmente em um PC } \\
\text { dedicado com conexão a VAN, e definissem o } \\
\text { mapeamento e regras de conexão para quaisquer } \\
\text { mensagens diferentes e ligação com parceiros }\end{array}$ & $\begin{array}{l}\text { Empresas instalam um pequeno cliente serviço web em } \\
\text { qualquer PC conectado à internet e somente define os } \\
\text { diretórios dos arquivos input e output }\end{array}$ \\
\hline \multirow{2}{*}{ 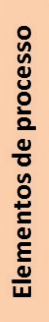 } & $\begin{array}{l}\text { Tempo de } \\
\text { instalação }\end{array}$ & $\begin{array}{l}\text { Devido à complexidade técnica, o tempo de instalação } \\
\text { levava várias semanas e exigia testes separados para } \\
\text { cada nova mensagem ou ligação com parceiro }\end{array}$ & $\begin{array}{l}\text { Tempo de instalação somente leva alguns dias, a não ser } \\
\text { que esforço extra seja exigido para importar e exportar } \\
\text { arquivos do ERP da empresa. Uma vez conectada, nenhum } \\
\text { esforço extra é exigido para conectar a outros parceiros } \\
\text { conectados }\end{array}$ \\
\hline & $\begin{array}{l}\text { Controle e } \\
\text { monitoramento de } \\
\text { processo }\end{array}$ & $\begin{array}{l}\text { O departamento interno de TI de cada empresa é } \\
\text { responsável por monitorar constantemente se as } \\
\text { mensagens EDI se traduziram corretamente e foram } \\
\text { enviadas/recebidas com sucesso. Em caso de } \\
\text { problemas, suporte técnico pelo vendedor do } \\
\text { software EDI poderia ser necessário }\end{array}$ & $\begin{array}{l}\text { Significativas economias de escala existem para o provedor } \\
\text { de serviço intermediário que faz o controle e } \\
\text { monitoramento do processo para todas as empresas. } \\
\text { Qualquer problema técnico é tratado internamente pelo } \\
\text { provedor de serviço }\end{array}$ \\
\hline \multirow{3}{*}{ 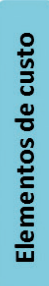 } & Custo de instalação & $\begin{array}{l}\text { A empresa tinha que pagar taxas significativas pelo } \\
\text { software EDI e pelo processo de instalação moroso e } \\
\text { complexo }\end{array}$ & $\begin{array}{l}\text { Taxa de instalação bem mais baixa ou ainda menor para o } \\
\text { mero serviço Web-EDI }\end{array}$ \\
\hline & $\begin{array}{l}\text { Custo da } \\
\text { infraestrutura da rede }\end{array}$ & $\begin{array}{l}\text { Taxa extra é paga pela assinatura anual à VAN e } \\
\text { baseada no volume de comunicação }\end{array}$ & Comunicação livre na internet \\
\hline & Custo corrente & $\begin{array}{l}\text { Custo de manutenção do software e custo extra } \\
\text { incorrido devido ao monitoramento técnico interno } \\
\text { necessários }\end{array}$ & $\begin{array}{l}\text { Taxa de serviço corrente baseada no número de transações, } \\
\text { somente cobrado do receptor da mensagem }\end{array}$ \\
\hline
\end{tabular}

Fonte: Adaptado de Pramatari (2007)

Segundo o VICS (2004), as práticas colaborativas, como o processo do CPFR, por exemplo, não estão fundamentalmente associadas a qualquer tecnologia. Entretanto, tais práticas dependem em grande parte de um suporte técnico capaz de facilitálas, o que está relacionado às ferramentas de apoio ao relacionamento baseado na tecnologia de informação, as quais procuram uma linguagem comum entre os agentes 
(VIVALDINO; PIRES; SOUZA, 2010). As aplicações de tecnologia de informação podem ajudar as organizações na tomada de decisão ao utilizar a tecnologia para reportar e acessar dados, assim como possibilitar aplicações analíticas, já que a gestão da cadeia de suprimentos, sem essa tecnologia, pode se tornar morosa e pouco fundamentada em dados e informações atualizados (FLIEDNER, 2003).

Segundo Vivaldino, Pires e Souza (2010), uma das forças trabalhadas pelas empresas tem sido o investimento em ferramentas tecnológicas e métodos que apoiam o relacionamento, para viabilizar as atividades colaborativas que buscam a melhoria do desempenho da cadeia de suprimentos. Chang et al. (2007) consideram os fatores críticos para o sucesso destas atividades a confiança entre os agentes da cadeia de suprimentos e a tecnologia empregada. Numa plataforma colaborativa, com base diária de informação, um fornecedor pode monitorar seus produtos, o nível de estoque e necessidade de reposição em cada ponto de venda (PRAMATARI; MILIOTIS, 2008). Assim, a tecnologia da informação, com destaque para a internet, possui papel fundamental em habilitar as organizações a desenvolver novas capacidades e habilidades que de outra forma seriam impossíveis de serem alcançadas, como a geração e disseminação de inteligência de mercado e a responsividade (VIVALDINO; PIRES; SOUZA, 2010).

\subsection{O papel facilitador das novas tecnologias de informação na integração das cadeias de suprimento}

O debate na literatura acerca da produtividade da tecnologia de informação ainda é instigado por diversos autores (SEGGIE; KIM; CAVUSGIL, 2006; PRAMATARI; 2007). De acordo com Handfield e Nichols (1999), com a emergência do computador pessoal e das redes de fibra ótica e com a explosão da internet e do World Wild Web, o custo e a disponibilidade de recursos de informação passaram a permitir ligações fáceis e a eliminar atrasos relacionados à informação nas cadeias de suprimentos, demonstrando o potencial das aplicações em tecnologia de informação para a integração dos fluxos nos processos interorganizacionais. Power (2005) comenta, em seu trabalho, que a noção de integração em cadeias de suprimento não é nova, mas que apenas recentemente tornouse viável que as empresas tenham acesso a informações acuradas, de forma rápida e acessível, o que se deve principalmente aos avanços em tecnologia de informação. Segundo o autor, a informação é o único elemento na cadeia de suprimentos que tem se tornado menos caro ao longo do tempo, possibilitando a evolução das práticas colaborativas entre membros da cadeia de suprimentos.

Seggie, Kim e Cavusgil (2006) investigam especificamente o domínio dos recursos de tecnologia de informação, constituídos pelo alinhamento da tecnologia de informação e pela integração dos sistemas interempresariais entre membros da cadeia de suprimentos, que eles consideraram mais susceptíveis a estarem incorporados nos processos gerenciais de uma empresa. Os autores defendem que a capacidade de uma empresa maximizar sua utilização de recursos de tecnologia de informação tem impacto 
no seu desempenho, já que o uso de um ativo particular seria mais importante que a mera existência do ativo em si. Tal argumentação vem amparada em pressupostos da visão baseada em recursos (Barney, 1991), de que um recurso pode trazer vantagem competitiva caso seja inimitável. Nesse sentido, a capacidade da empresa em maximizar o uso da tecnologia de informação demonstraria uma consciência objetiva de como esta poderia explorar recursos para assegurar uma integração mais intensa na cadeia de suprimentos.

Segundo Seggie, Kim e Cavusgil (2006), a capacidade do dono de um recurso receber o benefício igual ao valor criado por aquele recurso é definido como a apropriação do recurso. Assim, a apropriação da tecnologia de informação por uma empresa seria a capacidade desta em explorar o potencial do recurso de tecnologia de informação plenamente. Os autores defendem que quando uma empresa maximiza o uso de tecnologia de informação avançada para criar uma vantagem sobre seus concorrentes, a apropriação da tecnologia de informação provavelmente vai ser uma fonte de vantagem competitiva. Isso ocorre porque melhorias em tecnologia e em sua apropriação levam a um maior compartilhamento ativo de planejamento e das estratégias entre os parceiros, promovendo maior conectividade e integração, melhor comunicação e coordenação interorganizacional.

Paulraj, Lado e Chen (2008) afirmam que a tecnologia de informação tem um papel chave na redução de custos de coordenação interna e externa das empresas, e viabilizaria obtenção e troca de informações e conhecimento estratégico entre parceiros. Apesar de alguns autores defenderem que a tecnologia da informação é mais uma fonte de paridade competitiva que uma fonte de vantagem competitiva, outros autores afirmam que ela pode gerar vantagem competitiva sustentável na medida em que estiver incorporada nas rotinas e processos organizacionais para desenvolver capabilidades relacionais (PAULRAJ; LADO; CHEN, 2008; POWELL; DENT-MICALLEF, 1997). Baseado na lógica da visão relacional, a tecnologia da informação pode promover uma maior comunicação e servir como um mecanismo crítico para a ligação entre as empresas, o que pode gerar vantagens estratégicas duráveis para os parceiros da cadeia de suprimentos (DYER; SINGH, 1998; PAULRAJ; LADO; CHEN, 2008).

Pramatari (2007), ao analisar a evolução da tecnologia de informação no contexto da integração das cadeias de suprimento, afirma que a tecnologia emergente de serviços na web (web-services) e os padrões de interoperabilidade relacionados provêm respostas adequadas para transpor as maiores barreiras que tornavam os fornecedores receosos em participar de transações comerciais colaborativas: a incerteza técnica na confiabilidade de mercados eletrônicos e a complexidade de integrar sistemas de tecnologia de informação internos à plataforma colaborativa. Segundo a autora, os serviços na web ajudam a combinar os benefícios de custo e simplicidade da web com a independência e flexibilidade de uma solução descentralizada.

Uma arquitetura de aplicação descentralizada lida melhor com volumes de transação aumentados e alta frequência de interações entre parceiros comerciais, situação cada 
vez mais recorrente entre membros da cadeia de suprimentos. As implementações de RFID são exemplo de uma solução descentralizada. A autora ressalta, entretanto, que existe a necessidade de estabelecer claras práticas de trabalho de compartilhamento de informação e infraestruturas entre parceiros comerciais para suportar o uso de trocas de informação livres e baseadas em padrões. $O$ atendimento a estas exigências de práticas e infraestrutura é crítico para viabilizar os processos de negócio transformados, que foram facilitados pela tecnologia RFID (PRAMATARI, 2007).

Sobre os riscos associados à implementação de novas tecnologias de informação, como a tecnologia de serviços web, Pramatari (2007) adverte que ainda há muitos esforços de padronização que precisam ser concluídos e se tornarem amplamente aceitos antes que as interfaces entre os serviços web de diferentes organizações sejam completamente padronizadas e capazes de permitir a integração dos processos da cadeia de suprimentos em larga escala. A expectativa, segundo a autora, é a de que esta seja a principal barreira e um dos maiores riscos tecnológicos associados a esta abordagem. Por fim, mas não menos importante, também se espera que a governança da infraestrutura desempenhe papel crítico no sucesso da abordagem tecnológica selecionada para suportar os processos colaborativos na cadeia de suprimentos.

\section{CONSIDERAÇÕES FINAIS}

A partir da discussão acerca do papel da tecnologia de informação na implementação de práticas colaborativas na cadeia de suprimentos, promovida com base na literatura sobre o tema, algumas conclusões são destacadas. O posicionamento dos autores parece convergir no sentido de atribuir à tecnologia da informação o status de uma condição relevante à colaboração (como antecedente) ou mesmo facilitadora (na condição de elemento correlato) da colaboração entre parceiros na cadeia de suprimentos (LEEUW; FRANSOO, 2009; PAULRAJ; LADO; CHEN, 2008; POWER, 2005; PRAMATARI, 2007; RAMANATHAN; GUNASEKARAN; 2012). Esses papéis expressam, em síntese, o fato de que a implementação e o alinhamento das novas tecnologias de informação promovem a integração dos sistemas organizacionais entre os membros de uma cadeia, vê dinamizam várias dimensões da colaboração, como o compartilhamento de informação, a sincronização dos processos de tomada de decisão, o compartilhamento de recursos e a comunicação efetiva, por exemplo. Nesse sentido, a tecnologia de informação aparece como um fator crítico para o sucesso das iniciativas colaborativas e, consequentemente, do impacto destas iniciativas no desempenho das empresas.

Alguns autores levantam, entretanto, aspectos relacionados à adoção da tecnologia de informação que podem representar riscos para as empresas. Pramatari (2007) destaca a questão da adequação da abordagem tecnológica ao contexto e infraestrutura dos parceiros da cadeia de suprimentos, que é crítica para o sucesso das práticas colaborativas a serem implementadas. Além disso, a maturidade da tecnologia deve ser suficiente para que sua complexidade não seja uma barreira relevante à sua implementação e 
utilização. É válido ressaltar que diversos autores concordam que o investimento em tecnologia, por si só, não é suficiente para instigar e manter as iniciativas de colaboração entre empresas (MIN et al., 2005; KENT; MENTZER, 2003; VIVALDINO; PIRES; SOUZA, 2010). Aspectos intangíveis como a confiança entre os parceiros e o estabelecimento de relacionamentos de longo prazo são críticos para a gestão colaborativa da cadeia de suprimentos.

Este artigo, de natureza teórica e conceitual, apresentou diferentes variáveis e elementos analíticos que direta ou indiretamente tratam da relevância da TI na integração dos processos em cadeias de suprimento e de como as novas tecnologias podem favorecer a difusão e intensificação de práticas colaborativas em díades de relacionamento em tais cadeias.

Algumas questões de pesquisa, nesse sentido, poderiam ser exploradas em futuros estudos sobre o tema, como, por exemplo, quais seriam os aspectos de convergência e diferenciação das teorias da RBV, dos custos de transação e da visão relacional relativamente ao papel da TI no desenvolvimento de práticas colaborativas em cadeias de suprimento. Uma revisão também de modelos e suportes teóricos para a governança dos relacionamentos em díades e cadeias de suprimento, em virtude do desenvolvimento e implementação de práticas colaborativas mediadas pela $\mathrm{TI}$, poderiam também contribuir para adensar o conhecimento da área de operações sobre o assunto. Por sua vez, estudos dedicados à investigação dos efeitos, sobre o desempenho organizacional, da proximidade física e da "proximidade digital" (por conta da intensificação da TI nos processos interorganizacionais) poderiam ser justificados em função da relevância prática e científica do tema em um cenário de forte globalização comercial e, simultaneamente, internacionalização das cadeias produtivas.

\title{
COLLABORATIVE PRACTICES IN THE SUPPLY CHAIN AND THE ROLE OF NEW INFORMATION TECHNOLOGIES
}

\begin{abstract}
Different theories and models have been validated in recent years, in researches that were aimed at the understanding of the antecedents, correlates and consequences of collaborative practices on the performance of enterprises and supply chains. There is also empirical evidence that corroborates the importance of new information technologies for the advancement of collaborative practices and the integration processes in the context of supply chains. This article, theoretical and conceptual in nature, proposes to synthesize part of these contributions and progress by proposing a discussion of the role of new
\end{abstract}


information technologies in promoting collaborative practices as a multidimensional, complex and strongly influenced by inter-process integration construct.

Key-words: Collaborative practices. Information technologies. Supply chain Management.

\section{Referências}

ARSHINDER, K.; DESHMUKH, S. Supply chain coordination: perspectives, empirical studies and research directions. International Journal of Production Economics, v.115, n.2, pp.316-335, 2008.

ATTARAN, M.; ATTARAN, S. Collaborative supply chain management: the most promising practice for building efficient and sustainable supply chains. Business Process ManagementJournal, v. 13, n. 3, pp. 390-404, 2007.

BARNEY, J. B. Firm resources and sustained competitive advantage.Journal of Management, v.17, n.1, pp.99-120, 1991.

BARRAT, M. Understanding the meaning of collaboration in the supply chain. Supply Chain Management: An International Journal, v. 9, n. 1, pp. 30-42, 2004.

BARRAT, M.; OLIVEIRA, A. Exploiting the experience of collaborative planning initiatives. International Journal of Physical Distribution \& Logistics Management, v.31, n.4, pp.266-289, 2001.

BOWERSOX, D.; CLOSS, D.; STANK, T. 21 ${ }^{\text {st }}$ Century Logistics: Making Supply Chain Integration a Reality. East Lansing: Michigan State University and Council of Logistics Management, 1999.

CAO, M; ZHANG, Q. Supply chain collaboration: impact on collaborative advantage and firm performance. Journal of Operations Management, v.29, pp.163-180, 2011.

CHANG, T. et al. A study of an augmented CPFR model for the $3 \mathrm{C}$ retail industry.Supply Chain Management: An International Journal, v.12, n.3, pp. 200-209, 2007.

DIERICKX, I; COOL, K. Asset stock accumulation and competitive advantage.Management Science, v. 35, n. 12, pp. 1504-1511, dec. 1989.

DYER; J. H.; SINGH, H. The Relational View: Cooperative Strategy and Sources of Interorganizational Competitive Advantage. Academy of Management Review, v. 23, n. 4, pp. 660-679, oct. 1998. 
FLIEDNER, G. CPFR: an emerging supply chain tool. Industrial Management \& Data Systems, v.103, n.1, pp. 14-21, 2003.

FORSLUND, H; JONSSON, P.The impact of forecast information quality on supply chain performance.International Journal of Operation \& Production Management, v.27, n.1, pp. 90-107, 2007.

GAMEIRO, P.A.D. As Organizações em Rede. Lisboa: Universidade Lusófona de Humanidades e Tecnologias, 2005.

GCI. EPC: A Shared Vision for Transforming Business Process, Global Commerce Initiative. [S.I.:s.d.], 2005. Disponível em: www.gci-net.org. Acesso em: 28 maio 2012.

HANDFIELD, R.; NICHOLS, E. Introduction to Supply Chain Management.Englewood Cliffs: Prentice-Hall, 1999.

HASHIBA, L. A colaboração com fornecedores e clientes, e sua influência no desempenho da firma: uma análise empírica na indústria brasileira de embalagens. 2008. $198 \mathrm{f}$. Dissertação (Mestrado em Administração de Empresas)-Escola de Administração de Empresas, Fundação Getúlio Vargas, São Paulo, 2008.

KAUFMAN, R. Nobody wins until the consumer says, "I'll take it". Apparel Industry Magazine, v.58, n.3, pp.14-16, 1997.

KENT, J. L.; MENTZER, J. T. The effect of investment inter-organizational information technology in a retail supply chain. Journal of Business Logistics, v.24, n.2, pp. 155-175, 2003.

KETCHEN, D. J.; HULT, G. T. M. Bridging organization theory and supply chain management: The case of best value supply chains. Journal of Operations Management, v. 25, n. 2, pp. 573-580, 2007.

KRAUSE, D. R.; HANDFIELD, R. B.; TYLER, B. B.The relationships between supplier development, commitment, social capital accumulation and performance improvement. Journal of Operations Management, v. 25, p. 528-545, 2007.

LAVASSANI, K.; MOVAHEDI, B.; KUMAR, V. Evolution of Supply Chain Theories: A comprehensive literature review. Production and Operations Management Society (POMS), California, USA. 2008. Proceedings..., 2008.

LEE, S.; PAK, B.; LEE, H. Business value of B2B electronic commerce: the critical role of inter-firm collaboration. Electronic Commerce Research and Applications, v.2, pp. 350361, 2003.

LEEUW, S.; FRANSOO, J. Drivers of close supply chain collaboration: one size fits all? International Journal of Operations \& Production Management, v. 29, n. 7, pp. 720739, 2009. 
LI, S. et al. The impact of supply chain management practices on competitive advantage and organizational performance. Omega, v. 34, pp. 107-124, 2006.

MENTZER, J. T. et al. Defining supply chain management. Journal of Business Logistics, v. 22, n. 2, p. 1-25, 2001.

MIN, S. et al. Supply chain collaboration: what's happening? The International Journal of Logistics Management, v.16, n.2, pp.237-256, 2005.

NYAGA, G.; WHIPPLE, J.; LYNCH, D. Examining supply chain relationships: do buyer and supplier perspectives on collaborative relationships differ? Journal of Operations Management, v.28, n.2, pp.101-114, 2010.

PAULRAJ, A.; LADO, A.; CHEN, I. Inter-organizational communication as a relational competency: antecedents and performance outcomes in collaborative buyer-supplier relationships. Journal of Operations Management, v. 26, p. 45-64, 2008.

PIRES, S. Gestão da cadeia de suprimentos: conceitos, estratégias, práticas e casos. 1. ed. São Paulo: Atlas, 2004.

POWELL, T.; DENT-MICALLEF, A. Information technology as competitive advantage: the role of human, business, and technology resources. Strategic Management Journal, $v$. 18, n. 5, pp. 375-405, 1997.

POWER, D. Supply chain management integration and implementation: a literature review. Supply Chain Management: An International Journal, v.10, n.4, pp.252-263, 2005.

POWER, D.; SOHAL, A. Implementation and usage of electronic commerce in managing the supply chain: a comparative study of ten Australian companies. Benchmarking, v.9, n.2, pp.190-208, 2002.

PRAMATARI, K. Collaborative supply chain practices and evolvingtechnological approaches.Supply Chain Management: An International Journal, v.12, n.3, pp.210220, 2007.

PRAMATARI, K.; MILIOTIS, P.The impact of collaborative store ordering on shelf availability. Supply Chain Management: An International Journal, v. 13, n. 1, pp. 49-61, 2008.

RAMANATHAN, U; GUNASEKARAN, A. Supply chain collaboration: impact of success in long-term partnerships. [S.I.:s.d.], 2012. InternationalJournalofProductionEconomics. Disponível em: <http://dx.doi.org/10.1016/j.ijpe.2012.06.002>. Acesso em: 3 jun. 2012.

SEGGIE, S.; KIM, D; CAVUSGIL, S. Do supply chain IT alignment and supply chain interfirm system integration impact upon brand equity and firm performance? Journal of Business Research, v.59, pp.887-895, 2006. 
SEIFERT, D. Collaborative planning, forecasting, and replenishment: How to create a supply chain advantage. New York: American Management Association, 2003.

SHEFFI, Y. The value of CPFR. RIRL CONFERENCE, Lisbon, Portugal, 2002. Proceedings..., Lisboa, 2002.

SIMATUPANG, T. M.; SRIDHARAN, R.The collaborative supply chain.The International Journal of Logistics Management, v. 13, n. 1, pp. 15-30, 2002.

VOLUNTARY INTERINDUSTRY COMMERCE STANDARDS.VICS CPFR: An Overview. 2004. Disponível em: <http://www.vics.org/committees/cpfr/>. Acesso em: 18 jan. 2012.

VIVALDINO, M.; PIRES, S. R. I.; SOUZA, F. B. Importância dos fatores não-tecnológicos na implementação do CPFR. RAC, Curitiba, v. 14, n. 2, pp. 289-309, Mar./Abr., 2010.

WIENGARTEN, F. et al. Collaborative supply chain practices and performance: exploring the key role of information quality. Supply Chain Management: An International Journal, v. 15, n. 6, pp. 463-473, 2010.

WILLIAMSON, O. E. The Economic Institutions of Capitalism: Firms, Markets, Relational Contracting. New York, NY: Free Press, 1985.

ZAJAC, E. J.; OLSEN, C. P. From Transaction Cost to Transaction Value Analysis: Implications for the Study of Interorganizational Strategies. Journal of Management Studies, v. 30, n. 1, p. 131-145, jan. 1993. 


\section{SOBRE OS AUTORES}

\begin{tabular}{|l|l|} 
Engenheira de Produção graduada pela Universidade Federal de Minas Gerais \\
(UFMG), com aperfeiçoamento em Engenharia Industrial e de Gestão na \\
Universidade do Porto, Portugal. Mestranda em Administração no Centro de \\
Pós-Graduação e Pesquisas em Administração (CEPEAD) da UFMG, na área \\
de concentração de Gestão da Cadeia de Suprimentos e Operações. Seus \\
interesses de pesquisa estão focados em temas como gestão por processos de \\
negócio, gestão da cadeia de suprimentos, logística e pesquisa operacional no \\
âmbito da gestão de operaçães. Possui a certificação OMG Certified Expert in \\
BPM (OCEB), pelo Object Management Group (OMG), e desenvolve consultoria \\
em projetos de gestão de processos de negócio e modelagem de problemas, \\
de Castro
\end{tabular}

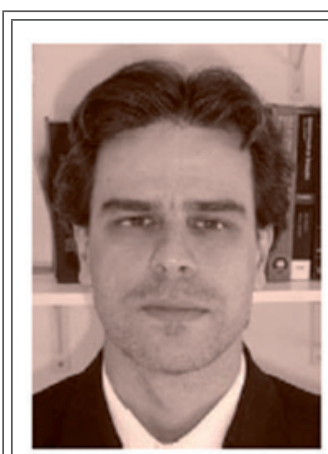

Marcelo Bronzo Ladeira
Docente associado do Departamento de Ciências Administrativas da Universidade Federal de Minas Gerais (UFMG) e um dos coordenadores do NIPELOG (Núcleo Interdisciplinar de Pesquisa e Extensão em Logística), vinculado ao Departamento de Ciências Administrativas da UFMG. Doutor e mestre em Administração pelo Centro de Pós-Graduação e Pesquisas em Administração (CEPEAD) da UFMG e foi pesquisador visitante na Faculdade de Economia e Comércio da Universidade de Bologna, Itália. Seus interesses de pesquisa estão focados em temas como melhores práticas nos processos logísticos de suprimento, manufatura e distribuição física; integração de processos-chave de negócios nos contextos da logística e do marketing empresarial; gestão de ambientes colaborativos na supplychain management; e modelos de maturidade para o gerenciamento de processos logísticos. 